\title{
Stokastik Talepli Kapasite Kısıtlı Araç Rotalama Problemine Yönelik Karşılaştırmalı Bir Yaklaşım
}

\author{
Çerkez Ağayeva ${ }^{1}$, Melis Alpaslan Takan ${ }^{2 *}$ \\ Geliş / Received: 18/04/2020 \\ Revize / Revised: 06/07/2020 \\ Kabul / Accepted: 06/07/2020
}

ÖZ

Bu çalışmada literatürde çalışılan en önemli kombinatoryal eniyileme problemlerinden biri olan stokastik araç rotalama problemi (SARP) ele alınmıştır. Bilindiği üzere klasik araç rotalama probleminde, araçların kapasiteleri ve müş̧erilerin talepleri bilinmektedir yani problem deterministiktir. Gerçek hayat problemlerinde problem parametreleri farklı durumlara göre değişkenlik gösterdiğinden, parametrelerin kesin değerlerinin bilinmesine az rastlanmaktadır. Bu yüzden belirtilen klasik araç rotalama probleminin belirsizlik koşulları altında formüle edilmesine ihtiyaç duyulmaktadır. Ele alınan çalışmada, müşteri taleplerinin belirsiz olduğu durumlar için, araç rotalama problemi analiz edilmiş̧tir ve talepler stokastik olarak modelde değerlendirilmiştir. Değişken talep durumlarını incelemek için düzgün, üstel ve Poisson olmak üzere 3 farklı dağılım kullanılarak, bu dağılımların problemin çözümleri üzerindeki etkileri incelenmiştir. Hesaplama sonuçları için GAMS yazılımı kullanılmıştır ve çalışmanın sonunda ele alınan problemin stokastik ve deterministik modellerinin sonuçları kıyaslanmıştır. 


\title{
A Comparative Approach to Capacitated Vehicle Routing Problem with Stochastic Demand
}

\begin{abstract}
In this study, stochastic vehicle routing problem (SVRP), which is one of the most important combinatorial optimization problems studied in the literature, is discussed. As it is known, the capacities of the vehicles and the demands of the customers are known in the classical vehicle routing problem, so the problem is deterministic. Since the problem parameters in real life problems vary according to the different situations, it is rare to know the exact values of the parameters. Therefore, there is a need to formulate the classical vehicle routing problem under uncertainty conditions. In this study, the vehicle routing problem is analyzed, and the demands are stochastically evaluated for the cases where customer demands are uncertain. In order to examine the variable demand conditions, the effects of these distributions on the solutions of the problem are investigated by using three different distributions, which are uniform, exponential, and Poisson. The GAMS software is used for computational results and the results of the stochastic and deterministic models of the problem are compared at the final part of the study.
\end{abstract}

Keywords- Vehicle Routing Problem, Uniform Distribution, Exponential Distribution, Poisson Distribution 


\section{I.GİRIŞ}

Araç rotalama problemi (ARP) ilk olarak Dantzig ve Ramser [1] tarafından kamyon dağıtım problemi olarak çalışılmış olup problemin amaç fonksiyonunda bütün müşterilerin taleplerini karşılayan en küçük maliyetli rotaların bulunması hedeflenmiştir. Literatürde NP-zor sınıfında sıklıkla çalışılan araç rotalama problemi türleri; klasik ARP, açık uçlu ARP, bölünmüş talepli ARP, zaman pencereli ARP, önce dağıt- sonra topla ARP, heterojen filolu ARP vb. [2]. Son zamanlarda klasik araç rotalama problemlerinin yanı sıra stokastik araç rotalama problemleri de incelenmektedir. Problemin parametrelerinin özellikleri ve farklı kısıtlar altında incelenmesi çeşitli stokastik araç rotalama problemlerini oluşturur.

Önceden belirtildiği gibi, klasik araç rotalama problemi deterministik bir problem olduğundan gerçek hayat ulaştırma ve lojistik problemlerindeki değişim durumlarını tam olarak yansıtamamaktadır. Belirsizlik durumları, neredeyse bütün gerçek hayat problemlerinde karşımıza çıkmaktadır. Bu yüzden problemdeki talep, mesafe ve zaman gibi bazı parametrelerin stokastik olarak ele alınması daha gerçekçi olmaktadır. Bu gibi stokastik durumlarda doğrusal programlama yöntemleri ele alınamamaktadır ve rassal değişkenlerin olduğu problemler ve buna bağlı gelişen stokastik ortam, stokastik programlama yaklaşımları ile ele alınarak çözülebilmektedir. Stokastik araç rotalama problemi günlük hayatta farklı alanlarda yer almaktadır. Örneğin; belediyelerin çöp toplama hizmetleri, insani yardım lojistiği, çay-kahve makinelerinin otomatlarının stoklarının belirlenmesi, farklı evlere yakıt dağıtımı vb.

SARP'nin en yaygın türü stokastik müşteri talepli araç rotalama problemidir [3,4] ve probleme yönelik literatürde farklı çalışmalar yer almaktadır. Örneğin Yang vd. [5] stokastik müşteri taleplerini yeniden stoklama üzerine; Smith vd. [6] ise çoklu araç önceliği ile stokastik talepli dinamik araç rotalama problemi üzerinde çalışmıştır. Bertsimas [7], stokastik talepli problemi, farklı rota güncelleme stratejileri ile ele almıştır. Tripathivd. [8] karınca tabanlı simülasyon ile stokastik talepli ARP'yi analiz etmiştir. Erera vd. [9] problemi süre kısıtları ile ele almıştır. Moghaddam vd. [10] probleme yönelik geliştirilmiş parçacık sürü eniyileme tekniğini kullanmıştır. Kenyon ve Morton [11] stokastik servis zamanlı ve yolculuk zamanlı problem üzerinde çalışmıştır. Goodson [12] çok bölmeli araç rotalama problemini çözebilmek için döngüsel düzenli tavlama benzetimi prosedürünü analiz etmiştir. Müşteri talepleri karşılanmadığında rotada hata oluştuğundan, tanımlı yenileme (recourse) hareketi (örneğin depoda ikmal gibi) yapılmalıdır Bu yüzden Bertsimas [13] ve Gendreau vd. [14] probleme yönelik iki aşamalı yöntemi uygulamışlardır. En yaygın yöntemler şans kısıtı programlama yöntemi ve yenileme ile stokastik programlama yöntemidir. Stokastik müş̧eri talepli ve stokastik servis zamanlı araç rotalama problemi için bir çalışma Campbell vd. [15] tarafından yapılmıştır. Laporte ise stokastik servis zamanı üzerinde yoğunlaşmıştır ve probleme yönelik kesin ve yaklaşık çözüm yöntemlerini analiz etmiştir [16]. Guo ve Mak, zaman pencereli stokastik araç rotalama problemi için bir sezgisel algoritma çalışmıştır [17]. Dinh vd.[18], müşteri taleplerinin stokastik olduğu araç rotalama problemleri için şans kısıtlı yöntemleri uygulamışlardır. Calvet vd. [19], heterojen filolustokastik araç rotalama problemini incelemişlerdir. Bu problemde, tüm araç türleri her müşteriye hizmet verememekte, stokastik müssteri talepleri bulunmakta ve asimetrik ağ yapısı vardır. Problemin çözümünde, tekrarlanan yerel arama algoritması ve Monte Carlo simülasyonu yöntemleri kullanılmıştır. Calvet vd. [20],çoklu depolu araç rotalama problemini ele almışlardır. Problemde, sınırlı depo kapasitesi ve stokastik müşteri talepleri bulunmaktadır.

SARP'nin sınıflandırılması yapılan çalışmalarda incelenmiş ve Tablo 1'de analiz edilmiştir [21]. Bu tabloya göre stokastik araç rotalama problemi farklı gruplara ayrışmaktadır. Birinci bölümde araç rotalama probleminde hangi kısmın stokastik olabileceği belirtilirken, ikinci bölümde stokastik problemin çözüm yöntemleri gösterilmiştir. Üçüncü kısımda ise grafik gösterim yani problemin ağ yapısından bahsedilmektedir. Eğer müşteriler arası mesafeler biliniyorsa ve $i^{\prime}$ 'den $j^{\prime} y e$ gidiş ile $j^{\prime}$ 'den $i^{\prime} y e$ gidiş mesafesi aynı ise problemin ağ yapısı simetrik, aynı değilse ağ yapısı asimetrik olarak adlandırılmaktadır. Tablonun dördüncü kısmında ise veri tipinden bahsedilmektedir. Kullanılan veriler, gerçek hayattan alınmış olabilir veya bir probleme göre uyarlama yapılmış veridir. Araç sayıları problemde tek olabilir (gezgin satıcı problemi), birden fazla olabilir ya da araç sayısı bilinmiyor olabilir. Araç kapasiteleri de probleme bağlı olarak kısıtlı ya da sonsuz kapasiteli olabilir. Bazı problemlerde ise kapasite bilinmeyebilir. Amaç fonksiyonları ise literatürde yine farklıllk gösteren bir diğer unsurdur. Örneğin; toplam maliyetin en küçüklenmesi, toplam mesafenin en küçüklenmesi, toplam rota zamanının en küçüklenmesi veya elde edilecek karın en büyüklenmesi gibi. Stokastik araç rotalama probleminde 
çalışılan yöntemler ise, şans kısıtlı programlama, dinamik programlama, markov zinciri, stokastik yenileme (stochastic with recourse) gibi yöntemlerdir.

Tablo 1. SARP'nin sınıflandırılması [21]

\begin{tabular}{|l|l|}
\hline 1 Stokastik Problem & $\mathbf{2}$ Çözüm \\
1.1 Stokastik müşteri & 2.1 Kesin \\
1.2 Stokastik müşteri talebi & 2.2 Sezgisel \\
1.3 Stokastik servis zamanı & 2.3 Diğer \\
1.4 Stokastik seyahat zamanı & \\
\hline 3 Grafik Gösterimi & $\mathbf{4}$ Veri tipi \\
3.1 Simetrik & 4.1 Gerçek veri \\
3.2 Asimetrik & 4.2 Sentez veri \\
3.3 Bilinmeyen & 4.3 Veri olmaması \\
\hline 5 Araç sayısı & 6 Araç kapasitesi \\
5.1 Tek araç & 6.1 Kapasite kisitlı \\
5.2 Çok araç & 6.2 Kapasite kisitsız \\
5.3 Bilinmeyen araç sayısı & 6.3 Bilinmeyen kapasite \\
& \\
\hline 7 Amaç fonksiyonu & $\mathbf{8}$ Uygulanan modeller \\
7.1 Enküçükleme & 8.1 Şans kisıtlı programlama \\
7.1.1 Maliyet enküçükleme & 8.2 Dinamik programlama \\
7.1.2 Uzaklik enküçükleme & 8.3 Markov zinciri \\
7.1.3 Zaman enküçükleme & 8.4 Stokastik yenileme (stochastic with recourse) \\
7.2 Enbüyükleme & \\
7.3 Diğer & \\
\hline
\end{tabular}

$\mathrm{Bu}$ çalışmada, stokastik talepli araç rotalama problemi ele alınmıştır. Belirsizliklerin kaynağı bu problem için talep olduğundan, bu belirsizlikler farklı dağılımlar ile ifade edilmiştir. Düzgün, üstel ve Poisson dağılım kullanılmış, farklı dağılımların problem üzerindeki etkileri gözlemlenmek istenmiştir. Böylece literatürde farklı çalışmalarda yapılan tek bir dağılım ile analiz etmenin yerine üç farklı dağılım kullanılarak probleme farklı bir bakış açısı sunulmuştur. Çalışmanın ikinci bölümünde araç rotalama probleminin matematiksel modeli detaylıca anlatılmaktadır. Üçüncü bölümde, GAMS programı kullanılarak elde edilen hesaplamalı sonuçlar verilmiştir. Çalışmanın son bölümünde ise sonuç ve önerilerden bahsedilmektedir.

\section{ARAÇ ROTALAMA PROBLEMININ MATEMATIKSEL MODELİ}

Literatürde ele alınan klasik araç rotalama problemi aşağıdaki şekildedir.

\section{Notasyon}

n: toplam müşteri sayıs1

$\mathrm{K}$ : toplam araç sayısı

$\mathrm{i}, \mathrm{j}, \mathrm{m}$ : müşteri indisi $\mathrm{i}, \mathrm{j}, \mathrm{m}=\{0, \ldots, \mathrm{n}\} .0$ depo noktası.

$\mathrm{k}:$ araç indisik $=\{1, \ldots, \mathrm{K}\}$

$\mathrm{d}_{\mathrm{i}}$ : i müş̧terisinin talep miktarı

$\mathrm{Q}_{\mathrm{k}}: \mathrm{k}$ aracının kapasitesi

$c_{i j}$ : i müşterisinden j müşterisine gitme maliyeti 


\section{Karar Değişkenleri}

$$
\mathrm{x}_{\mathrm{ijk}}:\left\{\begin{array}{rr}
1, & \text { eğer } \mathrm{k} \text { aracı i müşterisinden } \mathrm{j} \text { müşterisine giderse } \\
0, & \text { diğer durumda }
\end{array}\right.
$$

$\mathrm{u}_{\mathrm{i}}, \mathrm{u}_{\mathrm{j}}$ :alttur engelleme kısıtlarında kullanılan pozitif değişkenler

Bu gösterimler altında, problemin matematiksel modeli aşağıdaki gibidir:

\section{Amaç fonksiyonu}

$$
\mathrm{z}=\sum_{\mathrm{i}=0}^{\mathrm{n}} \sum_{\mathrm{j} \neq \mathrm{i}}^{\mathrm{n}} \sum_{\mathrm{k}=1}^{\mathrm{K}} \mathrm{c}_{\mathrm{ij}} \mathrm{x}_{\mathrm{ijk}}
$$

\section{Kısitlar}

$$
\begin{array}{lc}
\sum_{\mathrm{i}=0}^{\mathrm{n}} \sum_{\mathrm{j} \neq \mathrm{i}}^{\mathrm{n}} \mathrm{d}_{\mathrm{i}} \mathrm{x}_{\mathrm{ijk}} \leq \mathrm{Q}_{\mathrm{k}} \forall \mathrm{k} & \\
\sum_{\mathrm{i}=0}^{\mathrm{n}} \mathrm{x}_{\mathrm{imk}}-\sum_{\mathrm{j} \neq \mathrm{i}, \mathrm{x}}^{\mathrm{n}} \mathrm{x}_{\mathrm{k}}=0 & \mathrm{~m}=1, \ldots, \mathrm{n} ; \forall \mathrm{k} \\
\sum_{\mathrm{i}=0}^{\mathrm{n}} \sum_{\mathrm{k}=1}^{\mathrm{K}} \mathrm{x}_{\mathrm{ijk}}=1 & \mathrm{j} \neq \mathrm{i}=1, \ldots, \mathrm{n} \\
\mathrm{u}_{\mathrm{i}}-\mathrm{u}_{\mathrm{j}}+\mathrm{n} \sum_{\mathrm{k}=1}^{\mathrm{K}} \mathrm{x}_{\mathrm{ijk}} \leq \mathrm{n}-1 & \mathrm{i}=\ldots, \mathrm{n} ; \mathrm{j}=1, \ldots, \mathrm{n}, \mathrm{j} \neq \mathrm{i} \\
\mathrm{x}_{\mathrm{ijk}} \in\{0,1\} & \forall \mathrm{i}, \forall \mathrm{j}, \forall \mathrm{k}
\end{array}
$$

Modeldeki amaç fonksiyonu (1) toplam rota mesafesinin enküçüklenmesini ifade etmektedir. Kısıt (2) ise araç kapasite kısıtını yani rotanın toplam kapasitesinin, araç kapasitesini aşamayacağını ifade eder. Araç akış kısıtı olarak bilinen kısıt (3) ise şunu ifade etmektedir: eğer araç imüşterisindenmmüşsterisine giderse, aynı araç $m$ müşterisindenjmüş̧erisine (ya da depoya) gider. Problem, klasik rotalama problemi olduğundan, araçlar kapasiteleri dolduğunda depoya dönmek zorundadır. Kısıt (4) her müşteriye mutlaka ve sadece bir aracın uğraması gerektiğini belirtir. Miller vd. [22] tarafından geliştirilen kısıt (5) alttur engelleme kısıtıdır. (6). kısıt ise $0-1$ tamsayılı değişkenleri ifade eder.

\section{A. Kullanılan Yöntem}

Yukarıda incelenen klasik araç rotalama probleminin modelindeki $c_{i j}, d_{i}, Q_{k}$ parametreleri deterministiktir. Bu modelin ele aldığımız stokastik karşıllı̆ında, taleplerdeki belirsizlik durumlarını göz önünde bulundurmak için, talep değerleri rassal değişkenler ile ele alınmıştır. Çeşitli belirsizlik durumlarını dikkate almak için, talepler; düzgün, üstel ve Poisson dağılımı olmak üzere üç farklı yaklaşım ile analiz edilmiştir. Aşağıda bu dağılımların uygulama detaylarından bahsedilmektedir:

1) Düzgün dağıllm: Taleplerin homojen bölünmesine karşılık gelen durumları ifade eder ki, biz çalışmada en küçük talep ile en büyük talep $\left(\left[d_{\min }, d_{\max }\right]\right)$ aralığında tanımlanan yani olasılık yoğunluk fonksiyonu aşağıdaki şekilde olan rassal değişken ele alınmaktadır.

$$
f(x)= \begin{cases}0, & x \notin\left[d_{\text {min }}, d_{\text {max }}\right] \\ \frac{1}{d_{\max }-d_{\min }} & x \in\left[d_{\text {min }}, d_{\text {max }}\right]\end{cases}
$$

$X$ rassal değişkenin beklenen değeri $E(X)=\frac{d_{\min }+d_{\max }}{2}$, varyansı ise $\operatorname{Var}(X)=\frac{\left(d_{\max }-d_{\min }\right)^{2}}{12}$ şeklindedir.

2) Üstel dağıllım: Kuyruk teorisinde, sıralama problemlerindeki ardışık olayların incelenmesinde önemli rol oynayan üstel dağılımın parametresinin farklı değerleri, çeşitli gerçek durumlara karşılık gelmektedir. Bu problemde parametrenin doğru değerinin seçimi çok önemlidir. Bu yüzden farklı yaklaşımlar kullanılabilir. $\mathrm{Bu}$ çalışmada parametre olarak her bir test problem için taleplerin ortalama değerleri kullanılmıştır. Böylece 
$\lambda>0$ parametresi bulunan ortalamanın tersi olacak şekilde tanımlanmış olur ki bu rassal değişkenin beklenen değeri ve varyansı sirasıyla; $E(X)=\frac{1}{\lambda} \operatorname{ve} \operatorname{Var}(X)=\frac{1}{\lambda^{2}}$.

3) Poisson Dă̆glım: Sürekli zaman aralığında etkili olan üstel dağılımdan farklı olarak değil, kesikli zaman anlarında incelenen durumlar için genellikle Poisson dağılımı kullanılır. $\lambda>0$ parametresi ortalama taleplere göre bulunan Poisson dağılımının kütle fonksiyonu aşağıdaki gibidir:

$$
\mathrm{P}(\mathrm{X}=\mathrm{n})=\frac{\lambda^{\mathrm{n}} \mathrm{e}^{-\lambda}}{\mathrm{n} !}, n=0,1,2, \ldots
$$

Bu rassal değişkenin ortalama değeri ve varyansı: $(X)=\lambda, \operatorname{Var}(X)=\lambda$.

\section{HESAPLAMALI SONUÇLAR}

Modele belirsizliğin dahil olmasının sonuçlara etkisini analiz ederken yukarda belirtilen dağılım türleri için Christofides vd. [23] tarafından oluşturulan test problemleri ele alınmıştır. Bu veri setini kullanarak uygun stokastik modeller oluşturulmuş ve GAMS programı yardımı ile çözülmüştür. Parametrelerin seçimi sonuçları etkilediği için farklı test problemlerinin stokastik modele dönüştürülmesinde çeşitli yaklaşımlar kullanılabilir. Bahsedilen test problemlerinin müşteri koordinatları ve araç kapasiteleri alınarak müşteri talepleri farklı dağılımlar şeklinde (düzgün, üstel ve Poisson dağılımları) ele alınmıştır. Çalışmada 9 adet test problemi incelenmiş ve uygun dönüşümler yapılarak elde edilen hesaplama sonuçları Tablo 2'de verilmiştir.

Tablo 2.Dağılımlara göre test problemlerinin analizi

\begin{tabular}{|c|c|c|c|c|c|}
\hline Problem & $\begin{array}{c}\text { Ortalama Talep } \\
\text { Değerleri }\end{array}$ & $\begin{array}{l}\text { Deterministik } \\
\text { Çözüm }\end{array}$ & Düzgün Dağılım & Üstel Dağılım & Poisson Dağılım \\
\hline E016-05m & 16.125 & 345 & 345 & 259 & 238 \\
\hline E021-04m & 15.66 & 366 & 362 & 292 & 295 \\
\hline E022-06m & 1022.72 & 551 & 551 & 378 & 388 \\
\hline E023-03m & 443 & 568 & 568 & 470 & 470 \\
\hline E023-05m & 443 & 568 & 568 & 470 & 470 \\
\hline E026-08m & 14.11 & 859 & 840 & 685 & 714 \\
\hline
\end{tabular}

Tablo 2'deki ilk sütun hangi test probleminin çalışıldığını göstermektedir. İkinci sütun test problemlerinin ortalama müşteri taleplerini belirtmektedir. Bu ortalamalar her test problemi için hesaplanmıştır. Üçüncü sütun problemlerin deterministik (stokastiğe dönüştürülmemiş) çözümlerini belirtir. Sonraki sütunlar ise belirsizlik etkisi altında incelenen problemlerin çözümleridir. Birinci sütundaki test problemlerinin isimleri problemdeki müşteri ve araç adetlerini belirtmektedir (örneğin E016-03m test probleminde, 16 adet müşterive 3 adet araç olduğunu gösterir). Elde edilen çözümler bulunan en küçük toplam rota maliyetini belirtmektedir. Problemin deterministik halinde GAMS programının çalışma süresi her bir test problem için 2 saat verilmiştir. Çıkan sonuçlar 2 saatin sonuçlarına göre çıkan en iyi çözümlerdir. E016-03m test probleminde ortalama talep değerleri 16.125 'dir. Problemin deterministik çözümünde en iyi çözüm 278 olarak bulunmuştur. Düzgün dağılım ile problemin talep değerleri ele alındığında toplam rota maliyeti 278, üstel dağılım ile 238 ve Poisson dağılım ile 219 bulunmuştur. Böylece Poisson dağılım ile en küçük rota maliyeti elde edilmiştir. Müşteri taleplerinin Poisson dağılıma uyması diğer yöntemlere göre bu problemde daha avantajlıdır. E22-04m test problemine baktığımızda ise çıkan sonuçlara göre üstel dağılım diğerlerinden daha üstündür ve en küçük rota maliyeti 278 olarak bulunmuştur. E23-05m test probleminde ise üstel dağılım ve Poisson dağılım toplam rota maliyetini 470 olarak hesaplamıştır. Düzgün dağılım ise deterministik çözüm ile aynı değeri elde ederek 568 değerini bulmuştur. Bu test probleminde üstel dağılımın ve Poisson dağılımın çözüme etkisi aynı olmuştur. Tablo 2'den 
görüldüğü gibi genel olarak taleplerin düzgün dağlıma uyan stokastik karşllığı deterministik çözümle yakın sonuçlar vermektedir.

\section{SONUÇ VE ÖNERILER}

$\mathrm{Bu}$ çalışma kapsamında literatürde sıklıkla rastlanan stokastik talepli araç rotalama problemi ele alınmıştır. Günümüzde gerçek hayat problemlerinde, ani ve beklenmeyen değişimler ile çok fazla karşılaşıldığından, bu durumların ifade edilmesinde stokastik modellerin önemi büyüktür. Bu modellerde önceden önemsenmeyen veya umulmayan etkilerin dikkate alınması vurgulanmaktadır. Bu yüzden stokastik modeller deterministik modellere göre gerçek hayatı daha iyi yansıtmaktadır. Ele alınan problemin gerçek hayatta farklı uygulama alanları mevcuttur. Örneğin; benzin istasyonlarının arzı, çöp toplama arabalarının rotalanması, belirli bir zaman aralığında bir yoldan geçen arabaların sayısı, belirli bir zaman aralığında bir yoldan geçen müşterilerin sayısı vb.

Belirsizliklerin kaynağı farklı nedenler olduğu için bu belirsizlikler çeşitli dağılımlarla ifade edilir. Yukarıda da gösterildiği gibi klasik araç rotalama problemindeki farklı parametreler (zaman, yol, talep, kapsam vb.) gerçek hayatta tam ve kesin olarak bilinmemektedir ve bunlar belirtilen modelin çeşitli stokastik karşılığını doğurmaktadır. $\mathrm{Bu}$ çalışmada müşteri talepleri stokastik kabul edilerek ve üç farklı dağılıma uyarlanarak incelenmiştir. 9 farklı test problemi üzerinde GAMS programındaki CPLEX çözücüsü ile alınan sonuçlar kıyaslanmış ve analiz edilmiştir. Tablo 2 sonuçları analiz edilerek aşağıdaki bulgulara erişilmiştir:

- Taleplerin düzgün dağılımla ele alınmasıyla elde edilen sonuçları ile deterministik çözümleri birbiriyle benzemektedir.

- Müşteri sayısı fazla olduğunda tüm yaklaşımlar benzer sonuç vermektedir.

- Test problemlerine göre dağılımların üstünlükleri değişmektedir.

Bu sorunları analiz ederek aşağıdaki çözüm yaklaşımları geliştirilebilir:

- Taleplerin düzgün dağılıma farklı yaklaşımla simüle edilmesi gerekmektedir.

- Müşteri sayısının fazla olduğu problemlerde iki aşamalı çözüm yöntemleri denenebilir. Öncelikle benzer taleplere (talep noktalarının yakınlığı, taleplerin yakın değerleri vb.) göre farklı kümeleme yöntemleri kullanılarak sınıflandırma yapılmalı, daha sonra elde edilen bu sınıfların karakteristikleri göz önünde bulundurularak problem yeniden çözülebilir. Farklı sezgisel veya metasezgisel algoritmalar kullanılarak daha büyük boyutlu araç rotalama problemleri üzerinde çalışılabilinir.

- Çözüm yaklaşımlarının test problemlerine göre farklılı̆̆ının nedenleri araştırılmalı ve her bir dağılım için uygun problem türleri belirtilmelidir.

Gelecek çalışmalarda yukarıda belirtilen sorunları dikkate alarak stokastik araç rotalama probleminin daha detaylı incelenmesi hedeflenmektedir. Bunun yanı sıra farklı araç rotalama problem türleri (heterojen filolu, açık uçlu, bölünmüş talepli vb.) ele alınabilir ve her problem sınıfı için uygun yaklaşımlar belirlenebilir. İleriki çalışmalarda probleme süre kısıtları da eklenerek çeşitli analizler yapılabilir.

\section{KAYNAKLAR}

[1] Dantzig, G.B.,\& Ramser, J.H. (1959). The Truck Dispatching Problem. Informs, 6, 80-91.

[2] Toth, P., \& Vigo, D. (2002).The Vehicle Routing Problem, Bologna, SIAM.

[3] Dror M., \& Trudeau, P.. (1989). Savings by Split Delivery Routing. Transportation Science, 23, 141-145.

[4] Laporte, G., Louveaux, F., \& Mercure, H., (1989). Models and exact solutions for a class of stochastic locationrouting problems. European Journal of Operational Research, 39, 71-78.

[5] Yang, W-H., Kamlesh M., \& Ronald, B.H. (2000). Stochastic vehicle routing problem with restocking. Transportation Science, 34, 99-112. 
[6] Smith, S.L., Pavone, M., Bullos, F., \& Frazzoli, E. (2010). Dynamic vehicle routing with priority classes of stochastic demands. IAM Journal on Control and Optimization, 48(5), 3224-3245.

[7] Bertsimas, D. (1991). A vehicle routing problem with stochastic demand. Journal of Operations Research, 40(3), 554-585.

[8] Tripathi, M., Kuriger, G., Wan, H. (2009). An ant based simulation optimization for vehicle problem with stochastic demands. 2009 Winter Simulation Conference, December 13-16, Austin, USA.

[9] Erera, A.L., Morales, C.J., \& Savelsbergh, M. (2010). The vehicle routing problem with stochastic demand and duration constraints. Report, The Supply Chain and Logistics Institute, Georgia Institute of Technology School of Industrial and Systems Engineering Atlanta, GA.

[10] Moghaddam F.B., Babak, R.R., \& Sadjadi J.S. (2012). Vehicle routing problem with uncertain demands: An advanced particle swarm algorithm. Computers and Industrial Engineering, 62, 306-317.

[11] Kenyon, S.A., \& Morton, P.D. (2003). Stochastic vehicle routing with random travel times. Journal of Transportation Science, 37(1), 69-82.

[12] Goodson, J.C., Ohlmann, J.W., \& Thomas, B.W. (2012). Cyclic-order neighborhoods with application to the vehicle routing problem with stochastic demand. European Journal of Operational Research, 217(2), 312-323.

[13] Bertsimas, D. (1992). A vehicle routing problem with stochastic demand. Operations Research, 40, 574585.

[14] Gendreau, M., Laporte, G., \& Sèguin, R. (1996). Stochastic vehicle routing. European Journal of Operational Research, 88, 3-12.

[15] Campbell, M.A., Gendreau M., \& Thomas, B.W. (2011). The orienteering problem with stochastic travel and service Times. Annals of Operations Research, 186, 61-81.

[16] Laporte, G. (1992). The vehicle routing problem: An overview of exact and approximate algorithms. European Journal of Operational Research, 59, 345-358.

[17] Guo, Z., Mak, K.L. (2004). A heuristic algorithm for the stochastic vehicle routing problems with soft time windows. Proceedings of the 2004 Congress on Evolutionary Computation, IEEE, 19-23 June, Portland, USA.

[18] Dinh, T., Fukasawa, R., Luadtka, J. (2018). Exact algorithms for the chanceconstrained vehicle routing problem, Mathematical Programming, 172(1- 2), 105-138.

[19] Calvet, L., Bernaus, A.P., Travessat-Baro, O., Juan, A.A. (2016). A Simheuristic for the heterogeneous sitedependent asymetric VRP with stochastic demands, Advances in Artificial Intelligence, 408-417.

[20] Calvet, L.,Wang, D., Juan, A., Bove, L. (2019). Solving the multidepot vehicle routing problem with limited depot capacity and stochastic demands, International Transactions in Operational Research, 26, 459-484.

[21] Berhan, E., Beshah, B., Kitaw, D., \& Abraham, A. (2014). Stochastic vehicle routing problem: a literature survey. Journal of Information and Knowledge Management, 13 (3), 1450022:1-12. 


\begin{tabular}{|c|c|c|}
\hline & $\begin{array}{l}\text { BŞEÜ Fen Bilimleri Dergisi } \\
7(2), 971-979,2020\end{array}$ & $\begin{array}{r}\text { BSEU Journal of Science } \\
\text { DOI: } 10.35193 / \text { bseufbd.722677 }\end{array}$ \\
\hline $\begin{array}{l}\text { BILECIKSEYH EDEBALI } \\
\text { UNIVERSITES }\end{array}$ & & 58-7575 (http://dergipark.gov.tr/bseufbd) \\
\hline
\end{tabular}

[22] Miller, C. E., Tucker, A. W., \& Zemlin, R. A. (1960). Integer programming formulation of traveling salesman problems, Journal of the ACM, 7 (4), 326-329.

[23] Christofides, N., Mingozzi, A., \& Paolo, T. (1981). Exact algorithms for the vehicle routing problem, based on spanning tree and shortest path relaxations. Mathemtical Programming, 20 (1), 255-282. 